\title{
Processing XML Streams with Deterministic Automata
}

\author{
Todd J. Green ${ }^{1}$, Gerome Miklau ${ }^{2}$, Makoto Onizuka ${ }^{3}$, and Dan Suciu ${ }^{2}$ \\ 1 Xyleme SA, Saint-Cloud, France todd.green@xyleme.com \\ ${ }^{2}$ University of Washington Department of Computer Science \\ \{gerome, suciu\}@cs. washington. edu \\ 3 NTT Cyber Space Laboratories, NTT Corporation, oni@acm.org
}

\begin{abstract}
We consider the problem of evaluating a large number of XPath expressions on an XML stream. Our main contribution consists in showing that Deterministic Finite Automata (DFA) can be used effectively for this problem: in our experiments we achieve a throughput of about $5.4 \mathrm{MB} / \mathrm{s}$, independent of the number of XPath expressions (up to $1,000,000$ in our tests). The major problem we face is that of the size of the DFA. Since the number of states grows exponentially with the number of XPath expressions, it was previously believed that DFAs cannot be used to process large sets of expressions. We make a theoretical analysis of the number of states in the DFA resulting from XPath expressions, and consider both the case when it is constructed eagerly, and when it is constructed lazily. Our analysis indicates that, when the automaton is constructed lazily, and under certain assumptions about the structure of the input XML data, the number of states in the lazy DFA is manageable. We also validate experimentally our findings, on both synthetic and real XML data sets.
\end{abstract}

\section{Introduction}

Several applications of XML stream processing have emerged recently: contentbased XML routing [24], selective dissemination of information (SDI) [3, 6, 9], continuous queries [7], and processing of scientific data stored in large XML files $[13,25,19]$. They commonly need to process large numbers of XPath expressions (say 10,000 to 1,000,000), on continuous XML streams, at network speed.

For illustration, consider XML Routing [24]. Here a network of XML routers forwards a continuous stream of XML packets from data producers to consumers. A router forwards each XML packet it receives to a subset of its output links (other routers or clients). Forwarding decisions are made by evaluating a large number of XPath filters, corresponding to clients' subscription queries, on the stream of XML packets. Data processing is minimal: there is no need for the router to have an internal representation of the packet, or to buffer the packet after it has forwarded it. Performance, however, is critical, and [24] reports very poor performance with publicly-available tools. 
Our contribution here is to show that the lazy Deterministic Finite Automata (DFA) can be used effectively to process large numbers of XPath expressions, at guaranteed throughput. The idea is to convert all XPath expressions into a single DFA, then evaluate it on the input XML stream. DFAs are the most efficient means to process XPath expressions: in our experiments we measured a sustained throughput of about $5.4 \mathrm{MB} / \mathrm{s}$ for arbitrary numbers of XPath expressions (up to $1,000,000$ in our tests), outperforming previous techniques [3] by factors up to 10,000 . But DFAs were thought impossible to use when the number of XPath expressions is large, because the size of the DFA grows exponentially with that number. We analyze here theoretically the number of states in the DFA for XPath expressions, and consider both the case when the DFA is constructed eagerly, and when it is constructed lazily. For the eager DFA, we show that the number of label wild cards (denoted $*$ in XPath) is the only source of exponential growth in the case of a single, linear XPath expression. This number, however, is in general small in practice, and hence is of little concern. For multiple XPath expressions, we show that the number of expression containing descendant axis (denoted // in XPath) is another, much more significant source of exponential growth. This makes eager DFAs prohibitive in practice. For the lazy DFA, however, we prove an upper bound on their size that is independent of the number and shape of XPath expressions, and only depends on certain characteristics of the XML stream, such as the data guide [11] or the graph schema [1,5]. These are small in many applications. Our theoretical results thus validate the use of a lazy DFA for XML stream processing. We verify these results experimentally, measuring the number of states in the lazy DFA for several synthetic and real data sets. We also confirm experimentally the performance of the lazy DFA, and find that a lazy DFA obtains constant throughput, independent of the number of XPath expressions.

The techniques described here are part of an open-source software package ${ }^{4}$.

Paper Organization We begin with an overview in Sec. 2 of the architecture in which the XPath expressions are used. We describe in detail processing with a DFA in Sec. 3, then discuss its construction in Sec. 4 and analyze its size, both theoretically and experimentally. Throughput experiments are discussed in Sec. 5. We discuss implementation issues in Sec. 6, and related work in Sec 7. Finally, we conclude in Sec. 8.

\section{Overview}

\subsection{The Event-Based Processing Model}

We start by describing the architecture of an XML stream processing system [4], to illustrate the context in which XPath expressions are used. The user specifies several correlated XPath expressions arranged in a tree, called the query tree. An input XML stream is first parsed by a SAX parser that generates a stream of $S A X$ events (Fig. 1); this is input to the query processor that evaluates the

\footnotetext{
${ }^{4}$ Described in [4] and available at xmltk. sourceforge.net.
} 
XPath expressions and generates a stream of application events. The application is notified of these events, and usually takes some action such as forwarding the packet, notifying a client, or computing some values. An optional Stream Index (called SIX) may accompany the XML stream to speed up processing [4]: we do not discuss the index here.

The query tree, $Q$, has nodes labeled with variables and the edges with linear XPath expressions, $P$, given by the following grammar:

$$
P::=/ N|/ / N| P P \quad N::=E|A| \operatorname{text}(S) \mid *
$$

Here $E, A$, and $S$ are an element label, an attribute label, and a string constant respectively, and $*$ is the wild card. The function text(S) matches a text node whose value is the string S. While filters, also called predicates, are not explicitly allowed, we show below that they can be expressed. There is a distinguished variable, $\$ R$, which is always bound to the root. We leave out from our presentation some system level details, for example the fact that the application may specify under which application events it wants to receive the SAX events. We refer the reader to [4] for system level details.

Example 1. The following is a query tree (tags taken from [19]):

\begin{tabular}{|c|c|}
\hline$\$ D$ IN $\$ R /$ datas & \$H IN $\$ \mathrm{D} /$ history \\
\hline$\$$ T IN $\$ D /$ title & \$TH IN \$D/tableHead \\
\hline $\begin{array}{l}\text { \$N IN } \$ D / / \text { tableHead } / / * \\
\$ V \text { IN } \$ N / \text { text ("Galaxy") }\end{array}$ & $\$ F$ IN $\$$ TH/field \\
\hline
\end{tabular}

Fig. 2 shows this query tree graphically. Fig. 3 shows the result of evaluating this query tree on an XML input stream: the first column shows the XML stream, the second shows the SAX events generated by the parser, and the last column shows the application events.

Filters Currently our query trees do not support XPath expressions with filters (a.k.a. predicates). One can easily implement filters over query trees in a naive way, as we illustrate here on the following XPath expression:

\$X IN \$R/catalog/product[@category="tools"][sales/@price > 200]/quantity

First decompose it into several XPath expression, and construct the query tree $Q$ in Fig. 4. Next we use our query tree processor, and add the following actions. We declare two boolean variables, b1, b2. On a $\$ \mathrm{Z}$ event, set b1 to true; on a $\$ \mathrm{U}$ event test the following text value and, if it is $>200$, then set b2 to true. At the end of a $\$ \mathrm{Y}$ event check whether $\mathrm{b} 1=\mathrm{b} 2=$ true. This clearly implements the two filters in our example. Such a method can be applied to arbitrary filters and predicates, with appropriate bookkeeping, but clearly throughput will decrease with the number of filters in the query tree. Approaches along these lines are discussed in $[3,6,9]$. More advanced methods for handling filters include event detection techniques [20] or pushdown automata [21].

The Event-based Processing Problem The problem that we address is: given a query tree $Q$, preprocesses it, then evaluate it on an incoming XML 


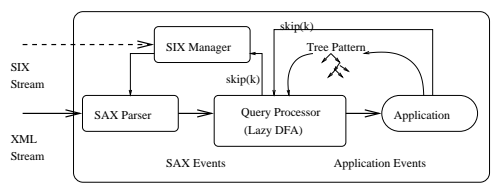

Fig. 1. System's Architecture

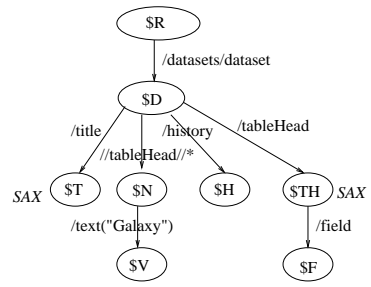

Fig. 2. A Query Tree

\begin{tabular}{|c|c|c|}
\hline $\begin{array}{l}\text { XML } \\
\text { Stream }\end{array}$ & $\begin{array}{l}\text { Parser } \\
\text { SAX Events }\end{array}$ & $\begin{array}{l}\text { Variable } \\
\text { Events }\end{array}$ \\
\hline$<$ datasets $\rangle$ & start (datasets) & start $(\$ R)$ \\
\hline Kdataset> & start (dataset) & start (\$D) \\
\hline Khistory> & start(history) & start $(\$ \mathrm{H})$ \\
\hline Kdate $\rangle$ & start (date) & \\
\hline $10 / 10 / 59$ & $\operatorname{text}(" 10 / 10 / 59 ")$ & \\
\hline K/date $>$ & end (date) & \\
\hline K/history $>$ & end(history) & end $(\$ \mathrm{H})$ \\
\hline Ktitle $>$ & start(title) & start $(\$ T)$ \\
\hline Ksubtitle $>$ & start(subtitle) & \\
\hline ptudy & text (Study) & \\
\hline $\mathrm{K} /$ subtitle $>$ & end(subtitle) & \\
\hline $\mathrm{K} /$ title $>$ & end(title) & \\
\hline & & end $(\$ \mathrm{~T})$ \\
\hline K/dataset $>$ & end(dataset) & end (\$D) \\
\hline & & \\
\hline K/datasets $>$ & end (datasets) & end $(\$ R)$ \\
\hline
\end{tabular}

Fig. 3. Events generated by a Query Tree

stream. The goal is to maximize the throughput at which we can process the $\mathrm{XML}$ stream. A special case of a query tree, $Q$, is one in which every node is either the root or a leaf node, i.e. has the form: $\$ \mathrm{x}_{1}$ in $\$ \mathrm{R} / \mathrm{e}_{1}, \$ \mathrm{X}_{2}$ in $\$ \mathrm{R} / \mathrm{e}_{2}, \ldots, \$ \mathrm{x}_{\mathrm{p}}$ in $\$ \mathrm{R} / \mathrm{e}_{\mathrm{p}}$ (each $e_{i}$ may start with // instead of /): we call $Q$ a query set, or simply a set. Each query tree $Q$ can be rewritten into an equivalent query set $Q^{\prime}$, as illustrated in Fig. 4.

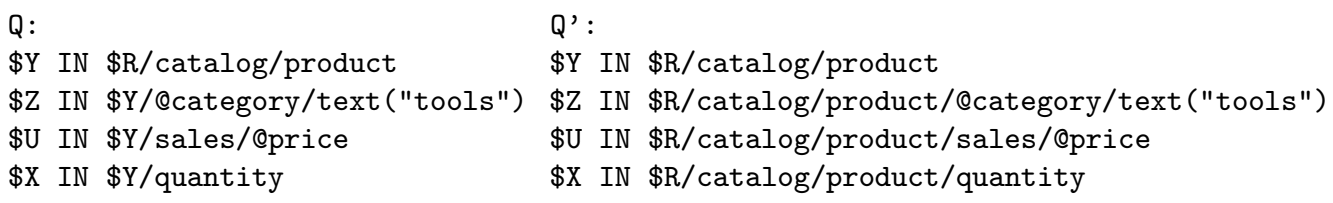

Fig. 4. A query tree $Q$ and an equivalent query set $Q^{\prime}$.

\section{Processing with DFAs}

\subsection{Background on DFAs}

Our approach is to convert a query tree into a Deterministic Finite Automaton (DFA). Recall that the query tree may be a very large collection of XPath expressions: we convert all of them into a single DFA. This is done in two steps: convert the query tree into a Nondeterministic Finite Automaton (NFA), then 
convert the NFA to a DFA. We review here briefly the basic techniques for both steps and refer the reader to a textbook for more details, e.g. [14]. Our running example will be the query tree $P$ shown in Fig. 5(a). The NFA, denoted $A_{n}$, is illustrated in Fig. 5(b). Transitions labeled $*$ correspond to $*$ or $/ /$ in $P$; there is one initial state; there is one terminal state for each variable $(\$ \mathrm{X}, \$ \mathrm{Y}, \ldots)$; and there are $\varepsilon$-transitions ${ }^{5}$. It is straightforward to generalize this to any query tree. The number of states in $A_{n}$ is proportional to the size of $P$.

Let $\Sigma$ denote the set of all tags, attributes, and text constants occurring in the query tree $P$, plus a special symbol $\omega$ representing any other symbol that could be matched by $*$ or $/ /$. For $w \in \Sigma^{*}$ let $A_{n}(w)$ denote the set of states in $A_{n}$ reachable on input $w$. In our example we have $\Sigma=\{a, b, d, \omega\}$, and $A_{n}(\varepsilon)=\{1\}$, $A_{n}(a b)=\{3,4,7\}, A_{n}(a \omega)=\{3,4\}, A_{n}(b)=\emptyset$.

The DFA for $P, A_{d}$, has the following set of states:

$$
\operatorname{states}\left(A_{d}\right)=\left\{A_{n}(w) \mid w \in \Sigma^{*}\right\}
$$

For our running example $A_{d}$ is illustrated ${ }^{6}$ in Fig. 5 (c). Each state has unique transitions, and one optional [other] transition, denoting any symbol in $\Sigma$ except the explicit transitions at that state: this is different from $*$ in $A_{n}$ which denotes any symbol. For example [other] at state $\{3,4,8,9\}$ denotes either $a$ or $\omega$, while [other] at state $\{2,3,6\}$ denotes $a, d$, or $\omega$. Terminal states may be labeled now with more than one variable, e.g. $\{3,4,5,8,9\}$ is labeled $\$ \mathrm{Y}$ and $\$ \mathrm{Z}$.

\subsection{The DFA at Run time}

Processing an XML stream with a DFA is very efficient. We maintain a pointer to the current DFA state, and a stack of DFA states. SAX events are processed as follows. On a start (element) event we push the current state on the stack, and replace the state with the state reached by following the element transition ${ }^{7}$; on an end (element) we pop a state from the stack and set it as the current state. Attributes and text (string) are handled similarly. No memory management is needed at run time ${ }^{8}$. Thus, each SAX event is processed in $O(1)$ time, and we can guarantee the throughput, independent of the number of XPath expressions. The main issue is the size of the DFA, which we discuss next.

\footnotetext{
${ }^{5}$ These are needed to separate the loops from the previous state. For example if we merge states 2,3 , and 6 into a single state then the $*$ loop (corresponding to $/ /$ ) would incorrectly apply to the right branch.

6 Technically, the state $\emptyset$ is also part of the DFA, and behaves like a "failure" state, collecting all missing transitions. We do not illustrate it in our examples.

7 The state's transitions are stored in a hash table.

8 The stack is a static array, currently set to 1024: this represents the maximum XML depth that we can handle.
} 


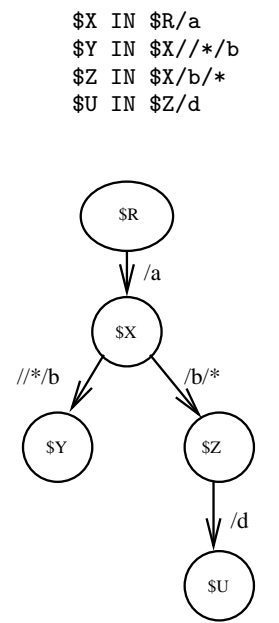

(a)

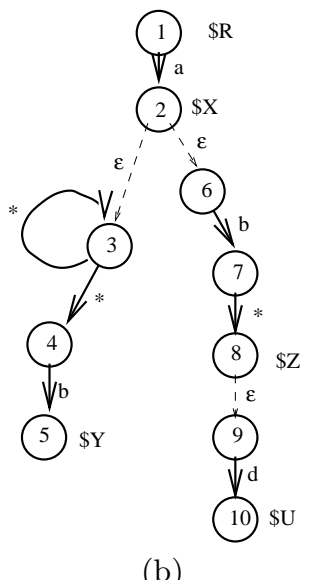

(b)

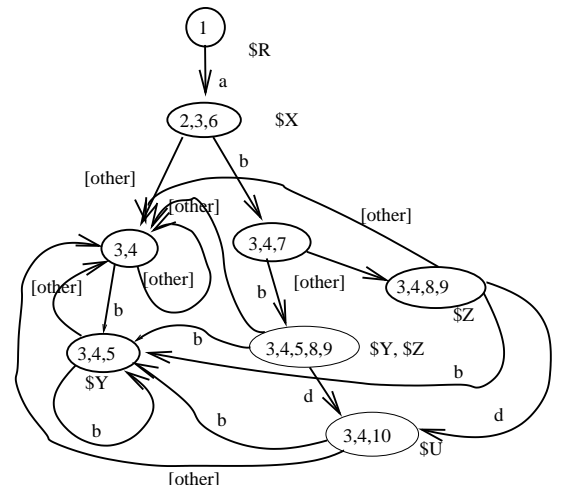

(c)

Fig. 5. (a) A query tree; (b) its NFA, $A_{n}$, and (c) its DFA, $A_{d}$.

\section{Analyzing the Size of the DFA}

For a general regular expression the size of the DFA may be exponential [14]. In our setting, however, the expressions are restricted to XPath expressions defined in Sec. 2.1, and general lower bounds do not apply automatically. We analyze and discuss here the size of the eager and lazy DFAs for such XPath expressions. We shall assume first that the XPath expressions have no text constants (text $(\mathrm{S})$ ) and, as a consequence, the alphabet $\Sigma$ is small, then discuss in Sec. 4.4 the impact of the constants on the number of states. As discussed at the end of Sec.2 we will restrict our analysis to query trees that are sets.

\subsection{The Eager DFA}

Single XPath Expression A linear XPath expression has the form $P=$ $p_{0} / / p_{1} / / \ldots / / p_{k}$ where each $p_{i}$ is $N_{1} / N_{2} / \ldots / N_{n_{i}}, i=0, \ldots, k$, and each $N_{j}$ is given by (1). We consider the following parameters:

$$
\begin{array}{ll}
k=\text { number of } / / \text { 's } & n_{i}=\text { length of } p_{i}, i=0, \ldots, k \\
m=\text { max \# of } * \text { 's in each } p_{i} \quad n=\text { length of } P, \sum_{i=0, k} n_{i} \\
s=\text { alphabet size }=|\Sigma| &
\end{array}
$$

For example if $P=/ / a / * / / a / * / b / a / * / a / b$, then $k=2\left(p_{0}=\varepsilon, p_{1}=a / *, p_{2}=\right.$ $a / * / b / a / * / a / b), s=3(\Sigma=\{a, b, \omega\}), n=9$ (node tests: $a, *, a, *, b, a, *, a, b)$, and $m=2$ (we have $2 *$ 's in $p_{2}$ ). The following theorem gives an upper bound on the number of states in the DFA, and is, technically, the hardest result in the paper. The proof is provided in the appendix. 


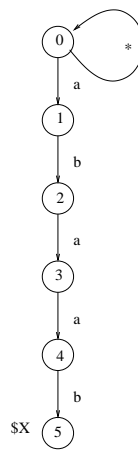

(a)

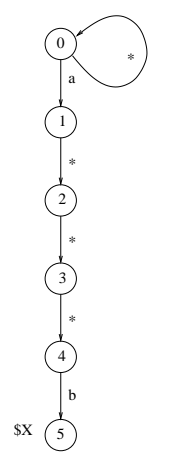

(c)

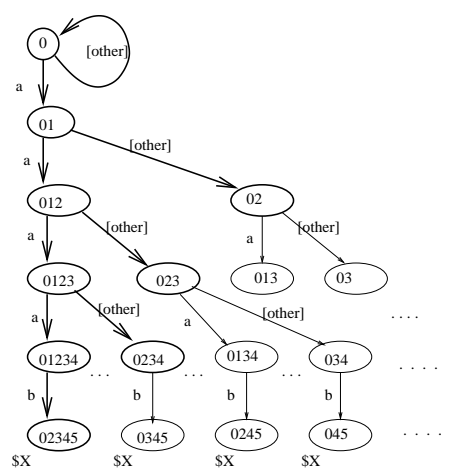

(d)

Fig. 6. The NFA (a) and the DFA (b) for $/ / a / b / a / a / b$. The NFA (c) and the DFA (with back edges removed) (d) for $/ / \mathrm{a} / * / * / * / \mathrm{b}$ : here the eager DFA has $2^{5}=32$ states, while the lazy DFA, assuming the DTD <!ELEMENT a $(a * \mid b)>$, has at most 9 states.

Theorem 1. Given a linear XPath expression $P$, define prefix $(P)=n_{0}$ and $\operatorname{suffix}(P)=k+k\left(n-n_{0}\right) s^{m}$. Then the eager DFA for $P$ has at most prefix $(P)+$ suffix $(P)$ states. In particular, when $k=0$ the DFA has at most $n$ states, and when $k>0$ the DFA has at most $k+k n s^{m}$ states.

We first illustrate the theorem in the case where there are no wild-cards $(m=0)$; then there are at most $k+k n$ states in the DFA. For example, if $p=/ / a / b / a / a / b$, then $k=1, n=5$ : the NFA and DFA shown in Fig. 6 (a) and (b), and indeed the latter has 6 states. This generalizes to $/ / N_{1} / N_{2} / \ldots / N_{n}$ : the DFA has only $n+1$ states, and is an isomorphic copy of the NFA plus some back transitions: this corresponds to Knuth-Morris-Pratt's string matching algorithm [8].

When there are wild cards $(m>0)$, the theorem gives an exponential upper bound. There is a corresponding exponential lower bound, illustrated in Fig. 6 (c), (d), showing that the DFA for $p=/ / a / * / * / * / b$, has $2^{5}$ states. It is easy to generalize this example and see that the DFA for $/ / a / * / \ldots / * / b$ has $2^{m+2}$ states $^{9}$, where $m$ is the number of $*$ 's.

Thus, the theorem shows that the only thing that can lead to an exponential growth of the DFA is the maximum number of $*$ 's between any two consecutive //'s. One expects this number to be small in most practical applications; arguably users write expressions like /catalog//product//color rather than /catalog//product/*/*/*/*/*/*/*/*/*/color. Some implementations of XQuery already translate a single linear XPath expression into DFAs [15].

Multiple XPath Expressions For sets of XPath expressions, the DFA also grows exponentially with the number expressions containing //. We illustrate first, then state the lower and upper bounds.

Example 2. Consider four XPath expressions:

\footnotetext{
${ }^{9}$ The theorem gives the upper bound: $1+(m+2) 3^{m}$.
} 

\$X1 IN $\$ R / /$ book//figure
\$X2 IN $\$ R / /$ table//figure
$\$ X 3$ IN $\$ R / /$ chapter//figure

The eager DFA needs to remember what subset of tags of \{book, table, chapter, note $\}$ it has seen, resulting in at least $2^{4}$ states. We generalize this below.

Proposition 1. Consider $p$ XPath expressions: $\$ \mathrm{X}_{1}$ IN $\$ \mathrm{R} / / \mathrm{a}_{1} / / \mathrm{b} \ldots$ $\$ \mathrm{X}_{\mathrm{p}}$ IN $\$ \mathrm{R} / / \mathrm{a}_{\mathrm{p}} / / \mathrm{b}$ where $a_{1}, \ldots, a_{p}, b$ are distinct tags. Then the DFA has at least $2^{p}$ states. ${ }^{10}$

Theorem 2. Let $Q$ be a set of XPath expressions. Then the number of states in the eager DFA for $Q$ is at most: $\sum_{P \in Q}(\operatorname{prefix}(P))+\prod_{P \in Q}(1+\operatorname{suffix}(P))$ In particular, if $A, B$ are constants s.t. $\forall P \in Q$, prefix $(P) \leq A$ and $\operatorname{suffix}(P) \leq B$, then the number of states in the eager DFA is $\leq p \times A+B^{p^{\prime}}$, where $p^{\prime}$ is the number of XPath expressions $P \in Q$ that contain //.

Recall that $\operatorname{suffix}(P)$ already contains an exponent, which we argued is small in practice. The theorem shows that the extra exponent added by having multiple XPath expressions is precisely the number of expressions with //'s. This result should be contrasted with Aho and Corasick's dictionary matching problem [2, $22]$. There we are given a dictionary consisting of $p$ words, $\left\{w_{1}, \ldots, w_{p}\right\}$, and have to compute the DFA for the set $Q=\left\{/ / w_{1}, \ldots, / / w_{p}\right\}$. Hence, this is a special case where each XPath expression has a single, leading //, and has no $*$. The main result in the dictionary matching problem is that the number of DFA states is linear in the total size of $Q$. Theorem 2 is weaker in this special case, since it counts each expression with a // toward the exponent. The theorem could be strengthened to include in the exponent only XPath expressions with at least two //'s, thus technically generalizing Aho and Corasick's result. However, XPath expressions with two or more occurrences of // must be added to the exponent, as Proposition 1 shows. We chose not to strengthen Theorem 2 since it would complicate both the statement and proof, with little practical significance.

Sets of XPath expressions like the ones we saw in Example 2 are common in practice, and rule out the eager DFA, except in trivial cases. The solution is to construct the DFA lazily, which we discuss next.

\subsection{The Lazy DFA}

The lazy DFA is constructed at run-time, on demand. Initially it has a single state (the initial state), and whenever we attempt to make a transition into a missing state we compute it, and update the transition. The hope is that only a small set of the DFA states needs to be computed.

This idea has been used before in text processing, but it has never been applied to such large number of expressions as required in our applications (e.g. 100,000): a careful analysis of the size of the lazy DFA is needed to justify its feasibility. We prove two results, giving upper bounds on the number of states

$\overline{10}$ Although this requires $p$ distinct tags, the result can be shown with only 2 distinct tags, and XPath expressions of depths $n=O(\log p)$, using standard techniques. 
in the lazy DFA, that are specific to XML data, and that exploit either the schema, or the data guide. We stress, however, that neither the schema nor the data guide need to be known in order to use the lazy DFA, and only serve for the theoretical results.

Formally, let $A_{l}$ be the lazy DFA. Its states are described by the following equation which should be compared to Eq.(2):

$$
\operatorname{states}\left(A_{l}\right)=\left\{A_{n}(w) \mid w \in \mathcal{L}_{\text {data }}\right\}
$$

Here $\mathcal{L}_{\text {data }}$ is the set of all root-to-leaf sequences of tags in the input XML streams. Assuming that the XML stream conforms to a schema (or DTD), denote $\mathcal{L}_{\text {schema }}$ all root-to-leaf sequences allowed by the schema: we have $\mathcal{L}_{\text {data }} \subseteq$ $\mathcal{L}_{\text {schema }} \subseteq \Sigma^{*}$.

We use graph schema $[1,5]$ to formalize our notion of schema, where nodes are labeled with tags and edges denote inclusion relationships. Define a simple cycle, $c$, in a graph schema to be a set of nodes $c=\left\{x_{0}, x_{1}, \ldots, x_{n-1}\right\}$ which can be ordered s.t. for every $i=0, \ldots, n-1$, there exists an edge from $x_{i}$ to $x_{i+1} \bmod n$. We say that a graph schema is simple, if for any two cycles $c \neq c^{\prime}$, we have $c \cap c^{\prime}=\emptyset$.

We illustrate with the DTD in Fig. 7, which also shows its graph schema [1]. This DTD is simple, because the only cycles in its graph schema (shown in Fig. 7 (a)) are self-loops. All non-recursive DTDs are simple. For a simple graph schema we denote $d$ the maximum number of cycles that a simple paths can intersect (hence $d=0$ for non-recursive schemes), and $D$ the total number of nonempty, simple paths: $D$ can be thought of as the number of nodes in the unfolding ${ }^{11}$. In our example $d=2, D=13$, and the unfolded graph schema is shown in Fig. 7 (b). For a query set $Q$, denote $n$ its depth, i.e. the maximum number of symbols in any $P \in Q$ (i.e. the maximum $n$, as in Sec. 4.1). We prove the following result in the appendix:

Theorem 3. Consider a simple graph schema with $d, D$, defined as above, and let $Q$ be a set of XPath expressions of maximum depth $n$. Then the lazy DFA has at most $1+D \times(1+n)^{d}$ states.

The result is surprising, because the number of states does not depend on the number of XPath expressions, only on their depths. In Example 2 the depth is $n=2$ : for the DTD above, the theorem guarantees at most $1+13 \times 3^{2}=118$ states in the lazy DFA. In practice, the depth is larger: for $n=10$, the theorem guarantees $\leq 1574$ states, even if the number of XPath expressions increases to, say, 100,000 . By contrast, the eager DFA has $\geq 2^{100000}$ states (see Prop. 1 ). Fig. 6 (d) shows another example: of the $2^{5}$ states in the eager DFA only 9 are expanded in the lazy DFA.

${ }_{11}$ The constant $D$ may, in theory, be exponential in the size of the schema because of the unfolding, but in practice the shared tags typically occur at the bottom of the DTD structure (see [23]), hence $D$ is only modestly larger than the number of tags in the DTD. 
Theorem 3 has many applications. First for non-recursive DTDs $(d=0)$ the lazy DFA has at most $1+D$ states $^{12}$. Second, in data-oriented XML instances, recursion is often restricted to hierarchies, e.g. departments within departments, parts within parts. Hence, their DTD is simple, and $d$ is usually small. Finally, the theorem also covers applications that handle documents from multiple DTDs (e.g. in XML routing): here $D$ is the sum over all DTDs, while $d$ is the maximum over all DTDs.

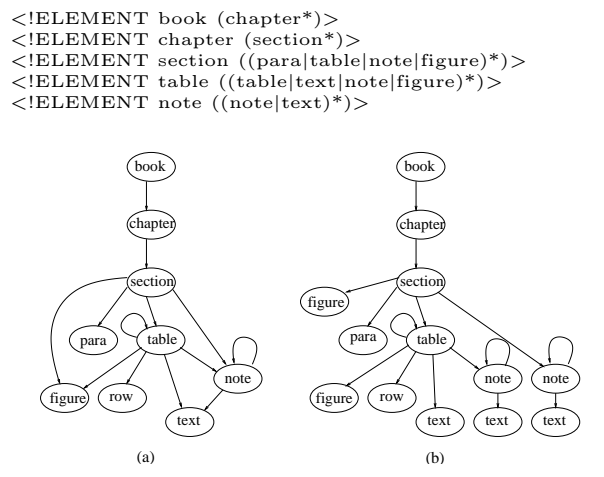

\begin{tabular}{||l|l|c|c||c||}
\hline \hline DTD & DTD & \multicolumn{2}{|c|}{ (DTD } & Data \\
Source & Names & Statistics) & size \\
\cline { 3 - 5 } & & $\begin{array}{c}\text { No. } \\
\text { elms }\end{array}$ & $\begin{array}{c}\text { Simple } \\
\text { MB }\end{array}$ & \\
\hline \hline [synthetic] & simple.dtd & 12 & Yes & - \\
\hline www. wapforum. org & prov.dtd & 3 & Yes & - \\
\hline www.ebxml.org & ebBPSS.dtd & 29 & Yes & - \\
\hline pir.georgetown.edu & protein.dtd & 66 & Yes & 684 \\
\hline xml.gsfc.nasa.gov & nasa.dtd & 117 & No & 24 \\
\hline UPenn Treebank & treebank.dtd & 249 & No & 56 \\
\hline
\end{tabular}

Fig. 8. Sources of data used in exFig. 7. A graph schema for a DTD (a) and periments. Only three real data its unfolding (b). sets were available.

The theorem does not apply, however, to document-oriented XML data. These have non-simple DTDs : for example a table may contain a table or a footnote, and a footnote may also contain a table or a footnote (hence, both $\{$ table $\}$ and $\{$ table,footnote $\}$ are cycles, and they share a node). For such cases we give an upper bound on the size of the lazy DFA in terms of Data Guides [11]. The data guide is a special case of a graph schema, with $d=0$, hence Theorem 3 gives:

Corollary 1. Let $G$ be the number of nodes in the data guide of an XML stream. Then, for any set $Q$ of XPath expressions the lazy DFA for $Q$ on that XML stream has at most $1+G$ states.

An empirical observation is that real XML data tends to have small data guides, regardless of its DTD. For example users occasionally place a footnote within a table, or vice versa, but do not nest elements in all possible ways allowed by the schema. All XML data instances described in [16] have very small data guides, except for Treebank [17], where the data guide has $G=340,000$ nodes.

$\overline{12}$ This also follows directly from (3) since in this case $\mathcal{L}_{\text {schema }}$ is finite and has $1+D$ elements: one for $w=\varepsilon$, and one for each non-empty, simple paths. 
Using the Schema or DTD If a Schema or DTD is available, it is possible to specialize the XPath expressions and remove all $*$ 's and //'s, and replace them with general Kleene closures: this is called query pruning in [10]. For example for the schema in Fig. 7 (a), the expression //table//figure is pruned to /book/chapter/section/(table)+/figure. This offers no advantage to computing the DFA lazily, and should be treated orthogonally. Pruning may increase the number of states in the DFA by up to a factor of $D$ : for example, the lazy (and eager) DFA for $/ / *$ has only one state, but if we first prune it with respect to a graph schema with $D$ nodes, the DFA has $D$ states.

Size of NFA tables A major component of the space used by the lazy DFA are the sets of NFA states that need to be kept at each DFA state. We call these sets NFA tables. The following proposition is straightforward, and ensures that the NFA tables do not increase exponentially:

Proposition 2. Let $Q$ be a set of $p$ XPath expressions, of maximum depths n. Then the size of each NFA table in the DFA for $Q$ is at most $n \times p$.

Despite the apparent positive result, the sets of NFA states are responsible for most of the space in the lazy DFA, and we discuss them in Sec. 6 .

\subsection{Validation of the Size of the Very Lazy DFA}

We ran experiments measuring the size of the lazy DFA for XML data for several publicly available DTDs, and one synthetic DTD. We generated synthetic data for these DTDs ${ }^{13}$. For three of the DTDs we also had access to real XML instances. The DTDs and the available XML instances are summarized in Fig. 8: four DTDs are simple, two are not; protein.dtd is non-recursive. We generated three sets of queries of depth $n=20$, with 1,000, 10,000, and 100,000 XPath expressions ${ }^{14}$, with $5 \%$ probabilities for both the $*$ and the $/ /$.

Fig. 9(a) shows the number of states in the lazy DFA for the synthetic data. The first four DTDs are simple, or non-recursive, hence Theorem 3 applies. They had significantly less states than the upper bound in the theorem; e.g. ebBPSS.dtd has 1058 states, while the upper bound is $12,790(D=29, d=$ $2, n=20$ ). The last two DTDs were not simple, and neither Theorem 3 nor Corollary 1 applies (since synthetic data has large data guides). In one case (Treebank, 100,000 expressions) we ran out of memory.

Fig. 9(b) shows the number of states in the lazy DFA for real data. This is much lower than for synthetic data, because real data has small dataguides, and Corollary 1 applies; by contrast, the dataguide for synthetic data may be as large as the data itself. The nasa.dtd had a dataguide with 95 nodes, less than the number of tags in the DTD (117) because not all the tags occurred in the data. As a consequence, the lazy DFA had at most 95 states. Treebank has a data guide with 340,000 nodes; the largest lazy DFA here had only 44,000 states.

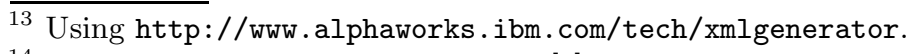

${ }^{14}$ We used the generator described in [9]. 

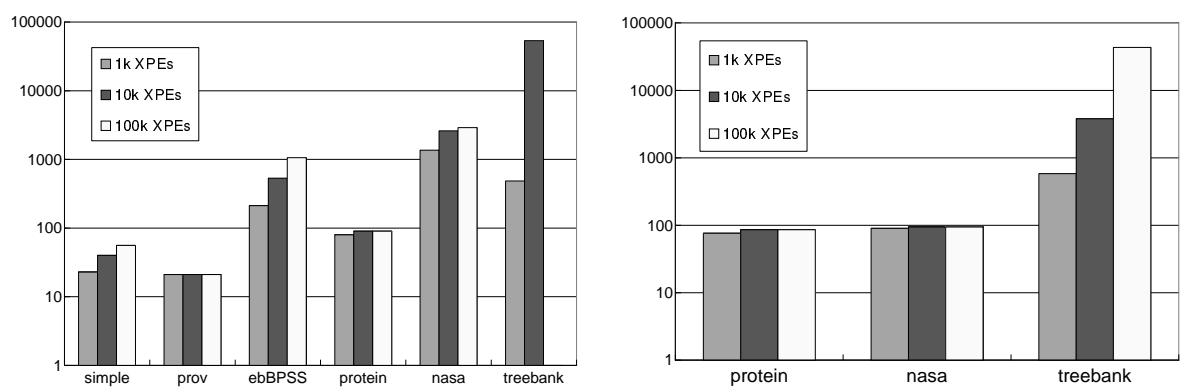

Fig. 9. Size of the lazy DFA for (left) synthetic data, and (right) real data. 1k means 1000 XPath expressions. For 100k XPath expressions for the treebank DTD with synthetic data we ran out of memory.

We also measured experimentally the average size of the NFA tables in each DFA state and found it to be around $p / 10$, where $p$ is the number of XPath expressions (graph shown in the appendix). Proposition 2 also gives an upper bound $O(p)$, but the constant measured in the experiments is much lower than that in the Theorem. These tables use most of the memory space and we address them in Sec. 6. Finally, we measured the average size of the transition tables per DFA state, and found it to be small (less than 40).

\subsection{Constant Values}

Finally, we comment on the impact of constant values on the number of states in the DFA. Each linear XPath expression can now end in a text (S) predicate, see Eq.(1). For a given set of XPath expressions, $Q$, let $\Sigma$ denote the set of all symbols in $Q$, including those of the form text(S). Let $\Sigma=\Sigma_{t} \cup \Sigma_{s}$, where $\Sigma_{t}$ contains all element and attribute labels and $\omega$, while $\Sigma_{s}$ contains all symbols of the form text(S). The NFA for $Q$ has a special, 2-tier structure: first an NFA over $\Sigma_{t}$, followed by some $\Sigma_{s}$-transitions into sink states, i.e. with no outgoing transitions. The corresponding DFA also has a two-tier structure: first the DFA for the $\Sigma_{t}$ part, denote it $A^{t}$, followed by $\Sigma_{s}$ transitions into sink states. All our previous upper bounds on the size of the lazy DFA apply to $A^{t}$. We now have to count the additional sink states reached by text $(\mathrm{S})$ transitions. For that, let $\Sigma_{s}=\left\{\operatorname{text}\left(\mathrm{S}_{1}\right), \ldots, \operatorname{text}\left(\mathrm{S}_{\mathrm{q}}\right)\right\}$, and let $Q_{i}, i=1, \ldots, q$, be the set of XPath expressions in $Q$ that end in text $\left(\mathrm{S}_{\mathrm{i}}\right)$; we assume w.l.o.g. that every XPath expression in $Q$ ends in some predicate in $\Sigma_{s}$, hence $Q=Q_{1} \cup \ldots \cup Q_{q}$. Denote $A_{i}$ the DFA for $Q_{i}$, and $A_{i}^{t}$ its $\Sigma_{t}$-part. Let $s_{i}$ be the number of states in $A_{i}^{t}, i=1, \ldots, q$. All the previous upper bounds, in Theorem 1, Theorem 3, and Corollary 1 apply to each $s_{i}$. We prove the following in the appendix.

Theorem 4. Given a set of XPath expressions $Q$, containing $q$ distinct constant values of the form text (S), the additional number of sink states in the lazy DFA due to the constant values is at most $\sum_{i=1, q} s_{i}$. 


\section{Experiments}

This section validates the throughput achieved by lazy DFAs in stream XML processing. Our execution environment consists of a dual $750 \mathrm{MHz}$ SPARC V9 with 2048MB memory, running SunOS 5.8. Our compiler is gcc version 2.95.2, without any optimization options.

We used the NASA XML dataset [19] and concatenated all the XML documents into one single file, which is about $25 \mathrm{MB}$. We generated sets of $1 \mathrm{k}(=1000)$, $10 \mathrm{k}, 100 \mathrm{k}$, and 1000k XPath expression using the XPath generator from [9], and varied the probability of $*$ and $/ /$ to $0.1 \%, 1 \%, 10 \%$, and $50 \%$ respectively. We report the throughput as a function of each parameter, while keeping the other two constant. For calibration and comparison we also report the throughput for parsing the XML stream, and the throughput of XFilter [3], which we re-implemented, without list balancing.

Figure 10 shows our results. In (a) we show the throughput as a function of the number of XPath expressions. The most important observation is that in the stable state (after processing the first $5-10 \mathrm{MB}$ of data) the throughput was constant, about $5.4 \mathrm{MB} / \mathrm{s}$. Notice that this is about half the parser's throughput, which was about $10 \mathrm{MB} / \mathrm{s}$; of course, the XML stream needs to be parsed, hence $10 \mathrm{MB} / \mathrm{s}$ should be seen as an upper bound on our platform. We observed in several other experiments with other datasets (not shown here) that the throughput is constant, i.e. independent on the number of XPath expressions. By contrast, the throughput of XFilter decreased linearly with the number of XPath expressions. The lazy DFA is about 50 times faster than XFilter on the smallest dataset, and about 10,000 times faster than XFilter on the largest dataset. Figure 10 (b) and (c) show the throughput as a function of the probability of $*$, and of the probability of // respectively.

The first 5MB-10MB of data in Fig. 10 represent the warm-up phase, when most of the states in the lazy DFA are constructed. The length of the warm-up phase depends on the size of the lazy DFA that is eventually generated. For the data in our experiments, the lazy DFA had the same number of states for $1 \mathrm{k}, 10 \mathrm{k}, 100 \mathrm{k}$, and $1000 \mathrm{k}$ (91, 95, 95, and 95 respectively). However, the size of the NFA tables grows linearly with the number of XPath expressions, which explains the longer tail: even if few states remain to be constructed, they slow down processing. In our throughput experiments with other datasets we observed that the lengths of the warm-up phase is correlated to the number of states in the lazy DFA.

\section{Implementation Issues}

Implementing the NFA tables In the lazy DFA we need to keep the set of NFA states at each DFA state: we call this set an NFA table. As shown in Prop. 2 the size of an NFA table is linear in the number of XPath expressions $p$, and about $p / 10$ in our experiments. Constructing and manipulating these tables during the warm-up phase is a significant overhead, both in space and in time. 


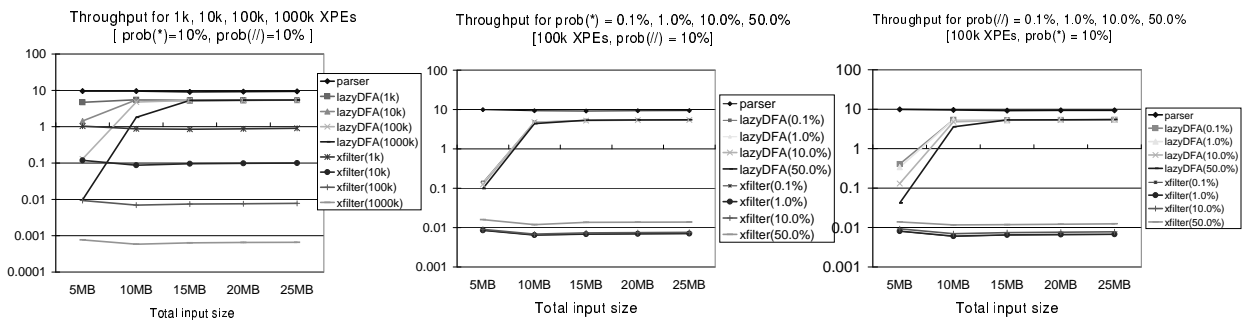

Fig. 10. Experiments illustrating the throughput of the DFA v.s. XFilter [3], as a function of the amount of XML data consumed. (left) varying number of XPath expressions $(1 \mathrm{k}=1000)$. (middle) varying probability of $*$. (right) varying probability of $/ /$.

We considered many alternative implementations for the NFA tables. There are three operations done on these sets: create, insert, and compare. For example a complex data set might have 10,000 DFA states, each containing a table of 30,000 NFA states and 50 transitions. Then, during warm-up phase we need to create $50 \times 10,000=500,000$ new sets; insert 30,000 NFA states in each set; and compare, on average, $500,000 \times 10,000 / 2$ pairs of sets, of which only 490,000 comparisons return true, the others return false. We found that implementing sets as sorted arrays of pointers offered the best overall performance. An insertion takes $O(1)$ time, because we insert at the end, and sort the array when we finish all insertions. We compute a hash value (signature) for each array, thus comparisons with negative answers take $O(1)$ in virtually all cases.

Optimizing space To save space, it is possible to delete some of the sets of NFA tables, and recompute them if needed: this may slow down the warm-up phase, but will not affect the stable state. It suffices to maintain in each DFA state a pointer to its predecessor state (from which it was generated). When the NFA table is needed, but has been deleted (a miss), we re-compute it from the predecessor's set; if that is not available, then we go to its predecessor, eventually reaching the initial DFA state for which we always keep the NFA table.

Updates Both online and offline updates to the set of XPath expressions are possible. In the online update, when a new XPath expression is inserted we construct its NFA, then create a new lazy DFA for the union of this NFA and the old lazy DFA. The new lazy DFA is very efficient to build (i.e. its warm-up is fast) because it only combines two automata, of which one is deterministic and the other is very small. When another XPath expression is inserted, then we create a new lazy DFA. This results in a hierarchy of lazy DFAs, each constructed from one NFA and another lazy DFA. A state expansion at the top of the hierarchy may cascade a sequence of expansions throughout the hierarchy. Online deletions are implemented as invalidations: reclaiming the memory used by the deleted XPath expressions requires garbage-collection or reference count. Offline updates can be done by a separate (offline) system, different from the production system. Copy the current lazy DFA, $A_{l}$, on the offline system, and also copy there the new query tree, $P$, reflecting all updates (insertions, deletions, etc). Then construct 
the eager DFA, $A_{d}$, for $P$, but only expand states that have a corresponding state in $A_{l}$, by maintaining a one-to-one correspondence from $A_{d}$ to $A_{l}$ and only expanding a state when this correspondence can be extended to the new state. When completed, $A_{d}$ is moved to the online system and processing resumes normally. The idea is that $A_{d}$ will be no larger than $A_{l}$ and, if there are only few updates, then $A_{d}$ will be approximately the same as $A_{l}$, meaning that the warm-up cost for $A_{d}$ is minimal.

\section{Related Work}

Two techniques for processing XPath expressions have been proposed. XFilter [3], its successor YFilter [9] and XTrie [6] evaluate large numbers of XPath expressions with what is essentially a highly optimized NFA. There is a space guarantee which is proportional to the total size of all XPath expressions. An optimization in XFilter, called list balancing can improve the throughput by factors of 2 to 4 . XTrie identifies common strings in the XPath expressions and organizes them in a Trie. At run-time an additional data structure is maintained in order to keep track of the interaction between the substrings. The throughput in XTrie is about 2 to 4 times higher than that in XFilter with list balancing.

In [20] the authors describe a technique for event detection. Events are sets of atomic events, and they trigger queries defined by other sets of events. The technique here is also a variation on the Trie data structure. This is an efficient event detection method that can be combined with lazy DFAs in order to process XPath expressions with filters.

Reference [15] describes a general-purpose XML query processor that, at the lowest level, uses an event based processing model, and show how such a model can be integrated with a highly optimized XML query processor. We were influenced by [15] in designing our stream processing model. Query processors like [15] can benefit from an efficient low-level stream processor. Specializing regular expressions w.r.t. schemes is described in $[10,18]$.

\section{Conclusion}

The challenge in fast XML stream processing with DFAs is that memory requirements have exponential bounds in the worst case. We proved useful theoretical bounds and validated them experimentally, showing that memory usage is manageable for lazy DFAs. We also validated lazy DFAs on stream XML data and found that they outperform previous techniques by factors of up to 10,000.

Acknowledgment We thank Peter Buneman, AnHai Doan, Ashish Gupta, Zack Ives, and Arnaud Sahuguet for their comments on earlier versions of this paper. Suciu was partially supported by the NSF CAREER Grant 0092955, a gift from Microsoft, and an Alfred P. Sloan Research Fellowship. 


\section{References}

1. S. Abiteboul, P. Buneman, and D. Suciu. Data on the Web : From Relations to Semistructured Data and XML. Morgan Kaufmann, 1999.

2. A. Aho and M. Corasick. Efficient string matching: an aid to bibliographic search. Communications of the ACM, 18:333-340, 1975.

3. M. Altinel and M. Franklin. Efficient filtering of XML documents for selective dissemination. In Proceedings of $V L D B$, pages 53-64, Cairo, Egipt, September 2000 .

4. I. Avila-Campillo, T. J. Green, A. Gupta, M. Onizuka, D. Raven, and D. Suciu. XMLTK: An XML toolkit for scalable XML stream processing. In Proceedings of PLANX, October 2002.

5. P. Buneman, S. Davidson, M. Fernandez, and D. Suciu. Adding structure to unstructured data. In Proceedings of the International Conference on Database Theory, pages 336-350, Deplhi, Greece, 1997. Springer Verlag.

6. C. Chan, P. Felber, M. Garofalakis, and R. Rastogi. Efficient filtering of XML documents with XPath expressions. In Proceedings of the International Conference on Data Engineering, 2002.

7. J. Chen, D. DeWitt, F. Tian, and Y. Wang. NiagaraCQ: a scalable continuous query system for internet databases. In Proceedings of the ACM/SIGMOD Conference on Management of Data, pages 379-390, 2000.

8. T. H. Cormen, C. E. Leiserson, and R. L. Rivest. Introduction to Algorithms. MIT Press, 1990.

9. Y. Diao, P. Fischer, M. Franklin, and R. To. Yfilter: Efficient and scalable filtering of xml documents. In Proceedings of the International Conference on Data Engineering, San Jose, California, February 2002.

10. M. Fernandez and D. Suciu. Optimizing regular path expressions using graph schemas. In Proceedings of the International Conference on Data Engineering, pages $14-23,1998$.

11. R. Goldman and J. Widom. DataGuides: enabling query formulation and optimization in semistructured databases. In Proceedings of Very Large Data Bases, pages 436-445, September 1997.

12. T. J. Green, G. Miklau, M. Onizuka, and D. Suciu. Processing xml streams with deterministic automata. Technical Report 02-10-03, University of Washington, 2002. Available from www.cs.washington.edu/homes/suciu.

13. D. G. Higgins, R. Fuchs, P. J. Stoehr, and G. N. Cameron. The EMBL data library. Nucleic Acids Research, 20:2071-2074, 1992.

14. J. Hopcroft and J. Ullman. Introduction to automata theory, languages, and computation. Addison-Wesley, 1979.

15. Z. Ives, A. Halevy, and D. Weld. An XML query engine for network-bound data. Unpublished, 2001.

16. H. Liefke and D. Suciu. XMill: an efficent compressor for XML data. In Proceedings of SIGMOD, pages 153-164, Dallas, TX, 2000.

17. M. Marcus, B. Santorini, and M.A.Marcinkiewicz. Building a large annotated corpus of English: the Penn Treenbak. Computational Linguistics, 19, 1993.

18. J. McHugh and J. Widom. Query optimization for XML. In Proceedings of VLDB, pages 315-326, Edinburgh, UK, September 1999.

19. NASA's astronomical data center. ADC XML resource page. http://xml.gsfc.nasa.gov/. 
20. B. Nguyen, S. Abiteboul, G. Cobena, and M. Preda. Monitoring XML data on the web. In Proceedings of the ACM SIGMOD Conference on Management of Data, pages 437-448, Santa Barbara, 2001.

21. D. Olteanu, T. Kiesling, and F. Bry. An evaluation of regular path expressions with qualifiers against XML streams. In Proc. the International Conference on Data Engineering, 2003.

22. G. Rozenberg and A. Salomaa. Handbook of Formal Languages. Springer Verlag, 1997.

23. A. Sahuguet. Everything you ever wanted to know about dtds, but were afraid to ask. In D. Suciu and G. Vossen, editors, Proceedings of WebDB, pages 171-183. Sringer Verlag, 2000.

24. A. Snoeren, K. Conley, and D. Gifford. Mesh-based content routing using XML. In Proceedings of the 18th Symposium on Operating Systems Principles, 2001.

25. J. Thierry-Mieg and R. Durbin. Syntactic Definitions for the ACEDB Data Base Manager. Technical Report MRC-LMB xx.92, MRC Laboratory for Molecular Biology, Cambridge,CB2 2QH, UK, 1992. 


\section{A Appendix}

\section{A.1 Proof of Theorem 1}

Proof. Let $A$ be the NFA for an XPath expression $P=p_{0} / / p_{1} / / \ldots / / p_{k}$ (see notations in Sec. 4.1). Its set of states, $Q$, has $n$ elements, in one-to-one correspondence with the symbol occurrences in $P$, which gives a total order on these states. $Q$ can be partitioned into $Q_{0} \cup Q_{1} \cup \ldots \cup Q_{k}$, which we call blocks, with the states in $Q_{i}=\left\{q_{i 0}, q_{i 1}, q_{i 2}, \ldots, q_{i n_{i}}\right\}$ corresponding to the symbols in $p_{i}$; we have $\sum_{i=0, k} n_{i}=n$. The transitions in $A$ are: states $q_{i 0}$ have self-loops with wildcards, for $i=1, \ldots, k$; there are $\varepsilon$ transitions from $q_{i-1 n_{i-1}}$ to $q_{i 0}, i=1, \ldots, k$; and there are normal transitions (labeled with $\sigma \in \Sigma$ or with wild-cards) from $q_{i(j-1)}$ to $q_{i j}$. Each state $\mathrm{S}$ in the DFA $A_{0}$ defined as $S=A(w)$ for some $w \in \Sigma^{*}$ $(S \subseteq Q)$, and we denote $S_{i}=S \cap Q_{i}, i=0,1, \ldots, k$. Our goal is to count the number of such states.

Before proving the theorem formally, we illustrate the main ideas on $P=$ $/ a / b / / a / * / a / / b / a / * / c$, whose NFA, $A$, is shown in Fig.11. Some possible states are:

$$
\begin{aligned}
& S_{1}=A(a)=\left\{q_{01}\right\} \\
& S_{2}=\left\{q_{10}, q_{12}\right\}=A(\text { a.b.e.e.a.b) } \\
& S_{3}=\left\{q_{10}, q_{12}, q_{20}, q_{21}\right\}=A(\text { a.b.e.e.a.b.a.e.e.a.b }) \\
& S_{4}=\left\{q_{10}, q_{11}, q_{20}, q_{22}\right\}=A(\text { a.b.e.e.a.b.a.e.e.b.a })
\end{aligned}
$$

The example shows that each state $S$ is determined by four factors: (1) whether it consists of states from block $Q_{0}$, like $S_{1}$, or from the other blocks, like $S_{2}, S_{3}, S_{4}$; there are $n_{0}$ states in the first category, so it remains to count the states in the second category only. (2) the highest block it reaches, which we call the depth: e.g. $S_{2}$ reaches block $Q_{1}, S_{3}, S_{4}$ reach block $Q_{2}$; there are $k$ possible blocks. (3) the highest internal state in each block it reaches: e.g. for $S_{3}$ the highest internal state is $q_{12}$, in block $Q_{1}$, while for $S_{4}$ the highest internal states is $q_{22}$, in $Q_{2}$ : there are at most $n-n_{0}$ choices here. (4) the particular values of the wildcards that allowed us to reach that highest internal state (not illustrated here); there are $s^{m}$ such choices.

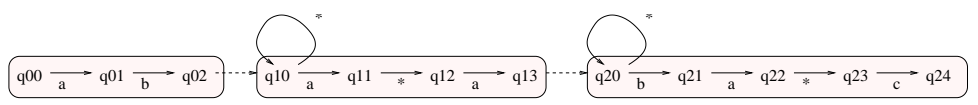

Fig. 11. The NFA for $/ a / b / / a / * / a / / b / a / * / c$.

We now make these arguments formal.

Lemma 1. Let $S=A(w)$ for some $w \in \Sigma^{*}$. If there exists some $q_{0 j} \in S$, for $j=0, \ldots, n_{0}-1$, then $S=\left\{q_{0 j}\right\}$. 
Proof. There are no loops at, and no $\varepsilon$ transitions to the states $q_{00}, q_{01}, \ldots, q_{0 j}$, hence we have $|w|=j$. Since there are no $\varepsilon$ transitions from these states, we have $S=A(w)=\left\{q_{0 j}\right\}$

This enables us to separate the sets $S=A(w)$ into two categories: those that contain some $q_{0 j}, j<n_{0}$, and those that don't. Notice that the state $q_{0 n_{0}}$ does not occur in any set of the first category, and occurs in exactly one set of the second category, namely $\left\{q_{0 n_{0}}, q_{10}\right\}$, if $k>0$ (because of the $\varepsilon$ transition between them), and $\left\{q_{0 n_{0}}\right\}$, if $k=0$ respectively. There are exactly $n_{0}$ sets $S$ in the first category. It remains to count the sets in the second category, and we will show that there are at most $k+k\left(n-n_{0}\right) s^{m}$ such sets, when $k>0$, and exactly one when $k=0$ : then, the total is $n_{0}+k+k\left(n-n_{0}\right) s^{m} \leq k+n k s^{m}$, when $k>0$, and is $n_{0}+1=n+1$ when $k=0$. We will consider only sets $S$ of the second kind from now on. When $k=0$, then the only such set is $\left\{q_{0 n_{0}}\right\}$, hence we will only consider the case $k>0$ in the sequel.

Lemma 2. Let $S=A(w)$. If $q_{d e} \in S$ for some $d>0$, then for every $i=1, \ldots, d$ we have $q_{i 0} \in S$.

Proof. This follows from the fact the automaton $A$ is linear, hence in order to reach the state $q_{d e}$ the computation for $w$ must go through the state $q_{i 0}$, and from the fact that $q_{i 0}$ has a self loop with a wild card.

It follows that for every set $S$ there exists some $d$ s.t. $q_{i 0} \in S$ for every $1 \leq i \leq d$ and $q_{i 0} \notin S$ for $i>d$. We call $d$ the depth of $S$.

Lemma 3. Let $S=A(w)$. If $q_{d e} \in S$ for some $d>0$, then for every $i=$ $1, \ldots, d-1$ and every $j \leq n_{i}$, if we split $w$ into $w_{1} . w_{2}$ where the length of $w_{2}$ is $j$, then $q_{i 0} \in A\left(w_{1}\right)$.

Proof. If the computation for $w$ reaches $q_{d e}$, then it must go through $q_{i 0}, q_{i 1}, \ldots, q_{i n_{i}}$. Hence, if we delete fewer than $n_{i}$ symbols from the end of $w$ and call the remaining sequence $w_{1}$ then the computation for $w_{1}$ will reach, and possible pass $q_{i 0}$, hence $q_{i 0} \in A\left(w_{1}\right)$ because of the selfloop at $q_{i 0}$.

We can finally count the maximum number of states $S=A(w)$. We fix a $w$ for each such $S$ (choosing one nondeterministically) and further associate to $S$ the following triple $\left(d, q_{t r}, v\right): d$ is the depth; $q_{t r} \in S$ is the state with the largest $r$, i.e. $\forall . q_{i j} \in S \Rightarrow j \leq r$ (in case when there are several choices for $t$ we pick the one with the largest $t$ ); and $v$ is the sequence of the last $r$ symbols in $w$. We claim that the triple $\left(d, q_{t r}, v\right)$ uniquely determines $S$. First we show that this claim proves the theorem. Indeed there are $k$ choices for $d$. For the others, we consider separate the choices $r=0$ and $r>0$. For $r=0$ there is a single choice for $q_{t r}$ and $v$ : namely $v$ is the empty sequence and $t=d$; for $r>0$ there are $n_{1}+n_{2}+\ldots+n_{d}<n-n_{0}$ choices for $q_{t r}$, and at most $s^{m}$ choices for $v$ since these correspond to choosing symbols for the wild cards on the path from $q_{t 1}$ to $q_{t r}$. The total is $\leq k+k\left(n-n_{0}\right) s^{m}$, which, as we argued, suffices to prove the theorem. It only remains to show that the triple $\left(d, q_{t r}, v\right)$ uniquely 
determines $S$. Consider two states, $S, S^{\prime}$, resulting in the same triples $\left(d, q_{t r}, v\right)$. We have $S=A(w . v), S^{\prime}=A\left(w^{\prime} . v\right)$ for some sequences $u, u^{\prime}$. It suffices to prove that $S \subseteq S^{\prime}$ (the inclusion $S^{\prime} \subseteq S$ is shown similarly). Let $q_{i j} \in S$. Clearly $i \leq d$ (by Lemma 1 ), and $j \leq r$. Decompose $v$ into $v_{1} \cdot v_{2}$, where $v_{2}$ contains the last $j-1$ symbols in $v$ : this is possible since $v$ 's length is $r \geq j$. Since $q_{i j} \in A(w . v)$ the last $j$ symbols in $w . v$ correspond to a transition from $q_{i 0}$ to $q_{i j}$. These last $j$ symbols belong to $v$, because $v$ 's length is $r \geq j$, hence we can split $v$ into $v_{1} \cdot v_{2}$, with the length of $v_{2}$ equal to $j$, and there exists a path from $q_{i 0}$ to $q_{i j}$ spelling out the word $v_{2}$. By Lemma 3 we have $q_{i 0} \in A\left(w^{\prime} . v_{1}\right)$ and, following the same path from $q_{i 0}$ to $q_{i j}$ we conclude that $q_{i j} \in A\left(u^{\prime} \cdot v_{1} \cdot v_{2}\right)=S^{\prime}$.

\section{A.2 Proof of Theorem 2}

Proof. Given a set of XPath expressions $Q$, one can construct its DFA, $A$, as follows. First, for each $P \in Q$, construct the DFA $A_{P}$. Then, $A$ is given by the product automaton $\prod_{P \in Q} A_{Q}$. From the proof of Theorem 1 we know that the states of $A_{P}$ form two classes: a prefix, which is a linear chain of states, with exactly prefix $(P)$ states, followed by a more complex structure with $\operatorname{suffix}(P)$ states. In the product automaton each state in the prefix of some $A_{P}$ occurs exactly once: these account for $\sum_{P \in Q} \operatorname{prefix}(P)$ states in $A$. The remaining states in $A$ consists of sets of states, with at most one state from each $A_{P}$ : there are at most $\prod_{P \in Q}(1+$ suffix $(P))$ such states.

\section{A.3 Proof of Theorem 3}

Proof. Given an unfolded graph schema $S$, the set of all root-to-leaf sequences allowed by $S, \mathcal{L}_{\text {schema }} \subseteq \Sigma^{*}$, can be expressed as:

$$
\mathcal{L}_{\text {schema }}=\{\varepsilon\} \cup \bigcup_{x \in \text { nodes }} \mathcal{L}_{\text {schema }}(x)
$$

where $\mathcal{L}_{\text {schema }}(x)$ denotes all sequences of tags up to the node $x$ in $S$. Our goal is to compute the number of states, as given by Eq.(3), with $\mathcal{L}_{\text {data }}$ replaced by $\mathcal{L}_{\text {schema }}$. Since the graph schema is simple and each simple path intersects at most $d$ cycles, we have:

$$
\begin{aligned}
\mathcal{L}_{\text {schema }}(x)= & \left\{w_{0} \cdot z_{1}^{m_{1}} \cdot w_{1} \ldots z_{d}^{m_{d}} \cdot w_{d} \mid\right. \\
& \left.m_{1} \geq 1, \ldots, m_{d} \geq 1\right\}
\end{aligned}
$$

where $w_{0}, \ldots, w_{d} \in \Sigma^{*}$ and $z_{1}, \ldots, z_{d} \in \Sigma^{+}$. (To simplify the notation we assumed that the path to $x$ intersects exactly $d$ cycles.) We use a pumping lemma to argue that, if we increase some $m_{i}$ beyond $n$ (the depth of the query set), then no new states are generated by Eq.(3). Let $u . z^{m} . v \in \mathcal{L}_{\text {schema }}(x)$ s.t. $m>n$. We will show that $A_{n}\left(u . z^{m} \cdot v\right)=A_{n}\left(u . z^{n} \cdot v\right)$. Assume $q \in A_{n}\left(u . z^{n} \cdot v\right)$. Following the transitions in $A_{n}$ determined by the sequence $u . z^{n} . v$ we notice that at least one $z$ in $z^{n}$ must follow a selfloop (since $n$ is the depth), corresponding to a // in one 
of the XPath expressions. It follows that $u \cdot a^{m} \cdot v$ has the same computation in $A_{n}$ : just follow that loop an additional number of times, hence $q \in A_{n}\left(u . z^{m} \cdot v\right)$. Conversely, let $q \in A_{n}\left(u . z^{m} . v\right)$ and consider the transitions in $A_{n}$ determined by the sequence $u . z^{m} . v$. Let $q^{\prime}$ and $q^{\prime \prime}$ be the beginning and end states of the $z^{m}$ segment. Since all transitions along the path from $q^{\prime}$ to $q^{\prime \prime}$ are either $z$ or wild cards, it follows that the distance form $q^{\prime}$ to $q^{\prime \prime}$ is at most $n$; moreover, there is at least one self-loop along this path. Hence, $z^{n}$ also determines a transition from $q^{\prime}$ to $q^{\prime \prime}$.

As a consequence, there are at most $(1+n)^{d}$ sets in $\left\{A_{n}(w) \mid w \in \mathcal{L}_{\text {schema }}(x)\right\}$ (namely corresponding to all possible choices of $m_{i}=0,1,2, \ldots, n$, for $i=$ $1, \ldots, d$ in Eq.(4)). It follows that there are at most $1+D(1+n)^{d}$ states in $A_{l}$.

\section{A.4 Proof of Theorem 4}

Proof. Each state in $A_{i}^{t}$ can have at most one transition labeled text $\left(\mathrm{S}_{\mathrm{i}}\right)$ : hence, the number of sink states in $A_{i}$ is at most $s_{i}$. The automaton for $Q=Q_{1} \cup \ldots \cup Q_{q}$ is can be described as the cartesian product automaton $A=A_{1} \times \ldots \times A_{q}$, assuming each $A_{i}$ has been extended with the global sink state $\emptyset$, as explained in Sec. 3.1. The $\Sigma_{s}$-sink states ${ }^{15}$ in $A$ will thus consists of the disjoint union of the $\Sigma_{s}$-sink states from each $A_{i}$, because the transitions leading to $\Sigma_{s}$-sink states in $A_{i}$ and $A_{j}$ are incompatible, when $i \neq j$. Hence, there are $\sum_{i} s_{i}$ sink states.

\section{A.5 Additional Experimental Results}
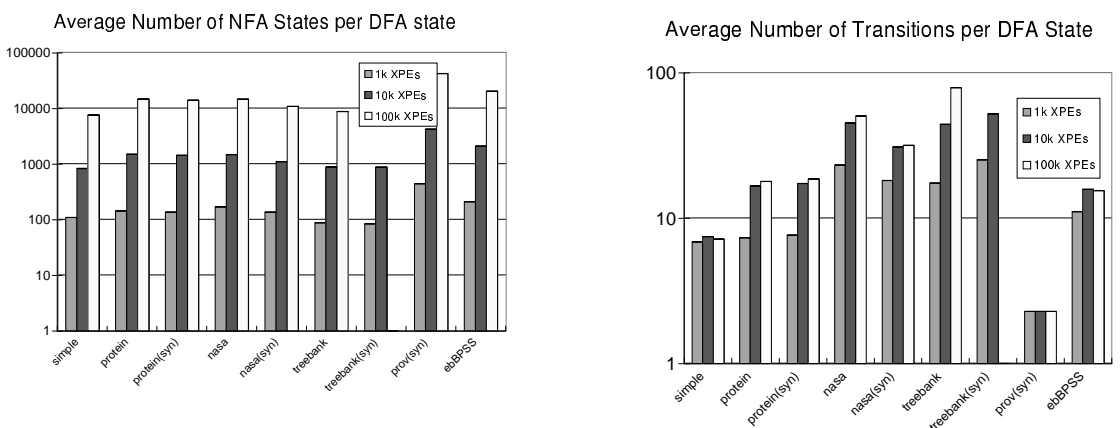

Fig. 12. Average size of the sets of NFA states, and average size of the transition table

Lazy DFA Size The two graphs in Figure 12 provide further experimental evidence on the size of the lazy DFA. Recall that each state in the lazy DFA is

\footnotetext{
${ }^{15}$ We call them that way in order not to confuse them with the $\emptyset$ sink state.
} 
identified by a set of states from the underlying NFA. Storing this list of states for each state in the lazy DFA is a major contributor to memory use in the system. The first graph shows that across our collection of datasets, the number of NFA states in a lazy DFA state averaged about $10 \%$ of number of XPath expressions evaluated.

Another measure of the complexity of the lazy DFA is the number of transitions per lazy DFA state. The second graph reports this averaged quantity for each dataset.

\section{Fallback Methods}

Memory Guarantees: Combined Processing Methods The upper bounds on the size of the lazy DFA given so far apply in most practical cases, but not in all cases. When they don't apply we can combine lazy DFAs with an alternative evaluation method, which is slower but has hard upper bounds on the amount of space used. Two such methods are xfilter [3] and xtrie [6], both of which process the XML stream as we do, by interpreting SAX events. Either can be used in conjunction with a lazy DFA so as to satisfy the following properties. (1) The combined memory used by the lazy DFA+fall-back is that of the fallback module plus a constant amount, $M$, determined by the user. (2) Most of the SAX events are processed only by the lazy DFA; at the limit, if the lazy DFA takes less than $M$ space, then it processes all SAX events alone. (3) Each SAX event is processed at most once by the lazy DFA and at most once by the fall-back module (it may be processed by both); this translates into a worst case throughput that is slightly less than that of the fall-back module alone.

We describe such a combined evaluation method that is independent of any particular fall-back method. To synchronize the lazy DFA and the fall-back module, we use two stacks of SAX events, $S_{1}$ and $S_{2}$, and a pointer $p$ to their longest common prefix. $S_{1}$ contains all the currently open tags: every start (element) is pushed into $S_{1}$, every end (element) is popped from $S_{1}$ (and the pointer $p$ may be decreased too). $S_{2}$ is a certain snapshot of $S_{1}$ in the past, and is updated as explained below; initially $S_{2}$ is empty. Start processing the XML stream with the lazy DFA, until it consumes the entire amount of memory allowed, $M$. At this point continue to run the lazy DFA, but prohibit new states from being constructed. Whenever a transition is attempted into a non-existing state, switch to the fall-back module, as follows. Submit end(element) events to the fall-back module, for all tags from the top of $S_{2}$ to the common-prefix pointer, $p$, then submit start (elements) from $p$ to the top of $S_{1}$. Continue normal operation with the fall-back module until the computation returns to the lazy DFA. At this point we copy $S_{1}$ into $S_{2}$ (we only need to copy from $p$ to the top of $S_{1}$ ), reset $p$ to the top of both $S_{1}$ and $S_{2}$, and continue operation in the lazy DFA (a depth counter will detect that). The global effect is that the lazy DFA processes some top part of the XML tree, while the fall-back module processes some subtrees: the two stacks are used to help the fall-back move from one subtree to the next.

We experimented with the treebank dataset, for which the lazy DFA is potentailly large, and found a 10/90 rule: by expanding only $10 \%$ of the states 
of the lazy DFA one can process $90 \%$ of all SAX events. Thus, only $10 \%$ need to be handled by the fall-back module. This is shown in Figure 13, which illustrates the number of SAX events processed by fallback methods when the lazy DFA is limited to a fixed number of states. The fixed number of DFA states is expressed as a percentage of the total required states for processing the input data. For example, when $100 \%$ of the lazy DFA states are allowed, then no SAX events are processed by fallback methods. The point at $12 \%$, for example, shows that with only $12 \%$ of the lazy DFA states most of the SAX events are handled by the DFA, and only a few (less than 10\%) by the fall-back method.

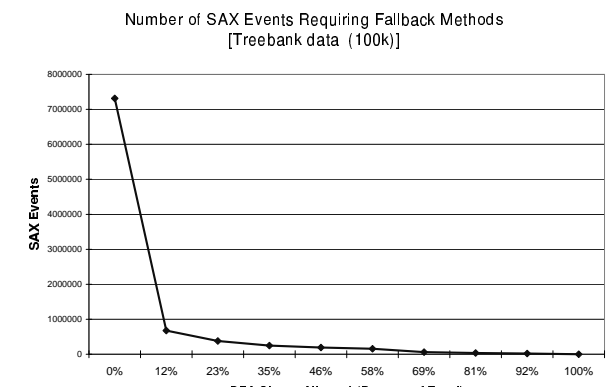

Fig. 13. Number of SA A $\mathrm{DF}$ events requiring fallback methods for lazy DFA limited to fixed number of states. 